\title{
An integrated analysis of hyponatremia in cancer patients receiving platinum-based or nonplatinum-based chemotherapy in clinical trials (JCOG1405-A)
}

\author{
Yasumasa Ezoe ${ }^{1}$, Junki Mizusawa², Hiroshi Katayama², Kozo Kataoka² and Manabu \\ Muto $^{1}$ \\ ${ }^{1}$ Department of Therapeutic Oncology, Kyoto University Graduate School of Medicine, Shogoin, Sakyo-ku, Kyoto 606-8507, \\ Japan \\ ${ }^{2}$ Japan Clinical Oncology Group (JCOG) Data Center/Operations Office, National Cancer Center Hospital, Tsukiji, Chuo-ku, \\ Tokyo 104-0045, Japan
}

Correspondence to: Manabu Muto, email: mmuto@kuhp.kyoto-u.ac.jp

Keywords: hyponatremia; platinum agent; cisplatin; carboplatin; risk factors

Abbreviations: JCOG: Japan Clinical Oncology Group; SIADH: syndrome of inappropriate antidiuretic hormone secretion; NCICTC: National Cancer Institute-Common Toxicity Criteria; Cl: confidence interval

Received: October 27, $2017 \quad$ Accepted: December 01, $2017 \quad$ Published: December 20, 2017

Copyright: Ezoe et al. This is an open-access article distributed under the terms of the Creative Commons Attribution License 3.0 (CC BY 3.0), which permits unrestricted use, distribution, and reproduction in any medium, provided the original author and source are credited.

\section{ABSTRACT}

Background: Hyponatremia is a common electrolyte abnormality in cancer patients who receive chemotherapy. Among anticancer agents, platinum-based agents are reported to cause chemotherapy-induced hyponatremia. However, the actual incidence and risk factors remain unknown.

Results: The reports of 29 trials were analyzed. The incidence of grade 3/4 hyponatremia was $11.9 \%$ in patients treated with platinum-based chemotherapy and $3.8 \%$ in those treated with nonplatinum-based regimens $(P<0.01)$. Univariable analysis revealed a high incidence of hyponatremia in patients receiving cisplatin, three-drug combination regimen, two-drug combination regimen with amrubicin or irinotecan, or high-dose cisplatin (weekly equivalent cisplatin dose $\geq 20 \mathrm{mg} / \mathrm{m}^{2}$ ), and in patients with small-cell lung cancer.

Conclusion: This is the first report of the actual incidence and the potential risk factors of chemotherapy-induced hyponatremia. Careful monitoring of serum sodium level is needed when platinum-based chemotherapy is administered.

Methods: This study included all clinical trials of systemic chemotherapies for solid cancers that were conducted by the Japan Clinical Oncology Group (JCOG) after January 2000 and of which the patient enrolment was completed by January 2014. The latest reports of each trial were used for analysis. The incidence of chemotherapy-induced grade 3/4 hyponatremia and the potential risk factors were investigated with univariable analysis.

\section{INTRODUCTION}

Hyponatremia is a common electrolyte disorder in cancer patients. However, recent studies suggest that hyponatremia might be a negative prognostic factor for cancer patients therefore its early detection and appropriate management might improve patient outcome [1-3]. The reported incidence of hyponatremia in cancer patients varies greatly according to the cancer type, clinical setting, and the serum sodium cutoff point, from $4 \%$ to $44 \%$ [4-7]. Hyponatremia in cancer patients can be caused by a number of factors including gastrointestinal losses, cardiac failure, diabetes insipidus, cancer-induced physiological changes or hormonal secretion, pulmonary diseases, central nervous system disorders, several drugs other 
than anticancer treatments (e.g., chlorpropamide, selective serotonin reuptake inhibitors, carbamazepine, antipsychotics, and vasopressin analogues), and several anticancer treatments $[8,9]$.

In clinical practice, chemotherapy-induced hyponatremia is an important adverse event because a rapid decrease in serum sodium level leads to disturbance of consciousness, convulsions, respiratory arrest, and treatment-related death in the worst-case scenario. In the event of chemotherapy-induced hyponatremia, chemotherapy may be discontinued or a dose reduction could be indicated. Currently, no effective prevention methods have been found; therefore, the only effective approach to avoid serious hyponatremia is early detection and appropriate treatment [7].

Among several anticancer agents, vinca alkaloids, alkylating agents, and platinum compounds are reported to be the chemotherapeutic agents that cause hyponatremia [7, 10]. Of these, platinum compounds are positioned as one of the key drugs used to treat major solid cancers, and are therefore frequently used in many chemotherapy regimens. As a result, platinuminduced hyponatremia is experienced more frequently than that associated with other chemotherapeutic agents in clinical practice. However, most of the previous reports were case studies and the exact incidence and risk factors of platinum-induced hyponatremia remain uncertain [11].

In this study, to evaluate the actual incidence and the potential risk factors of platinum-induced hyponatremia, we performed an integrated analysis of hyponatremia in patients with solid cancers receiving platinum-based or nonplatinum-based chemotherapy using the data from completed clinical trials conducted by the Japan Clinical Oncology Group (JCOG) [12-39].

\section{RESULTS}

\section{Study selection}

We identified 54 potentially relevant trials. All study protocols were screened, and 25 trials were excluded. The selection process and reasons for exclusion are detailed in Figure 1. A total of 29 trials were included in the final analysis.

\section{Study, patients, and treatment characteristics}

The reports of 29 phase II and III trials including 44 treatment arms (platinum-based chemotherapy, 27 treatment arms; nonplatinum-based chemotherapy, 17 treatment arms) were analyzed. The characteristics of each trial are summarized in Table 1A and 1B. The included trials consisted of three phase I/II trials (only the data of the phase II section were evaluated in this study), 10 phase II trials, one feasibility study, and 15 phase III trials. The target malignancies of included trials were non-small-cell lung cancer (6 trials), esophageal cancer (5), gastric cancer (5), small-cell lung cancer (3), ovarian, fallopian tube and peritoneal cancer (3), cervical cancer (2), colorectal cancer (1), pancreatic cancer (1), breast cancer (1), head and neck cancer (1), and bladder cancer (1).

\section{Incidence of platinum-based or nonplatinum- based chemotherapy-induced hyponatremia}

There was heterogeneity in the incidence of grade $3 / 4$ hyponatremia among patients receiving platinumbased chemotherapy, ranging from $2.6 \%$ to $29.1 \%$ in the 27 included treatment arms (2,238 patients). The incidence of grade 3/4 hyponatremia in patients receiving platinum-based chemotherapy $(11.9 \%)$ was significantly higher than that in patients receiving nonplatinum-based regimens $(3.8 \% ; P<0.01)$. Similarly, the incidence of grade 4 hyponatremia in patients receiving platinum-based chemotherapy $(1.5 \%)$ was significantly higher than that in patients receiving nonplatinum-based regimens $(0.4 \%$; $P<0.01$ ) (Figure 2). All trials in this analysis reported no grade 5 hyponatremia and no serious aftereffects related to hyponatremia.

\section{The potential risk factors of platinum-induced hyponatremia}

To evaluate the association between platinum and hyponatremia, we performed univariable analysis (Table 2).

\section{Influence of the type of platinum agent}

The incidence of grade 3/4 hyponatremia was greater in the cisplatin arm $(13.5 \%)$ than the carboplatin $\operatorname{arm}(7.6 \%)$. The difference between these subgroups was statistically significant $(P<0.01)$. A similar tendency was observed in the analysis of grade 4 hyponatremia (1.9\% in the cisplatin arm vs $0.3 \%$ in the carboplatin arm; $P<0.01)$.

\section{Influence of the platinum agent administration method}

The incidence of grade 3/4 hyponatremia in the bolus arm was $12.1 \%$, and that in the split arm was $11.4 \%$; there was no significant difference between these subgroups $(P=0.71)$. A similar tendency was observed in the analysis of grade 4 hyponatremia $(1.7 \%$ in the bolus arm vs $1.0 \%$ in the split arm; $P=0.32$ ).

\section{Influence of cisplatin dosage}

The incidence of grade 3/4 hyponatremia was greater in the high dosage arm (15.7\%) than the low dosage arm $(12.2 \%)$; however, there was no significant difference between these subgroups $(P=0.06)$. The incidence of grade 4 hyponatremia was significantly greater with high dosage $(2.9 \%)$ than low dosage $(1.4 \% ; P=0.04)$. 


\section{Influence of the number of concomitant medications}

The incidence of grade 3/4 hyponatremia was highest in the three-drug combination regimen arm $(21.4 \%)$, followed by the two-drug combination regimen $(11.8 \%)$, monotherapy (7.3\%), and four-drug combination (4.9\%). The differences among these subgroups were significant $(P<0.01)$; however, the incidence of hyponatremia in the four-drug combination was lowest among four subgroups, and a Cochran-Armitage trend test did not show statistical significance $(P=.45$; onesided test) (Figure 3 ). The incidence of grade 4 hyponatremia showed a similar tendency to that of grade $3 / 4$ hyponatremia.

\section{Influence of the type of concomitant medication}

The incidence of grade 3/4 hyponatremia was highest in the amrubicin combination regimen $(22.1 \%)$, followed by irinotecan (18.3\%), fluorouracil (11.8\%), taxane $(9.4 \%)$, and vinorelbine $(2.6 \%)$. The differences among these subgroups were significant $(P<0.01)$. The incidence of grade 4 hyponatremia showed a similar tendency to that of grade $3 / 4$ hyponatremia $(P<0.01)$.

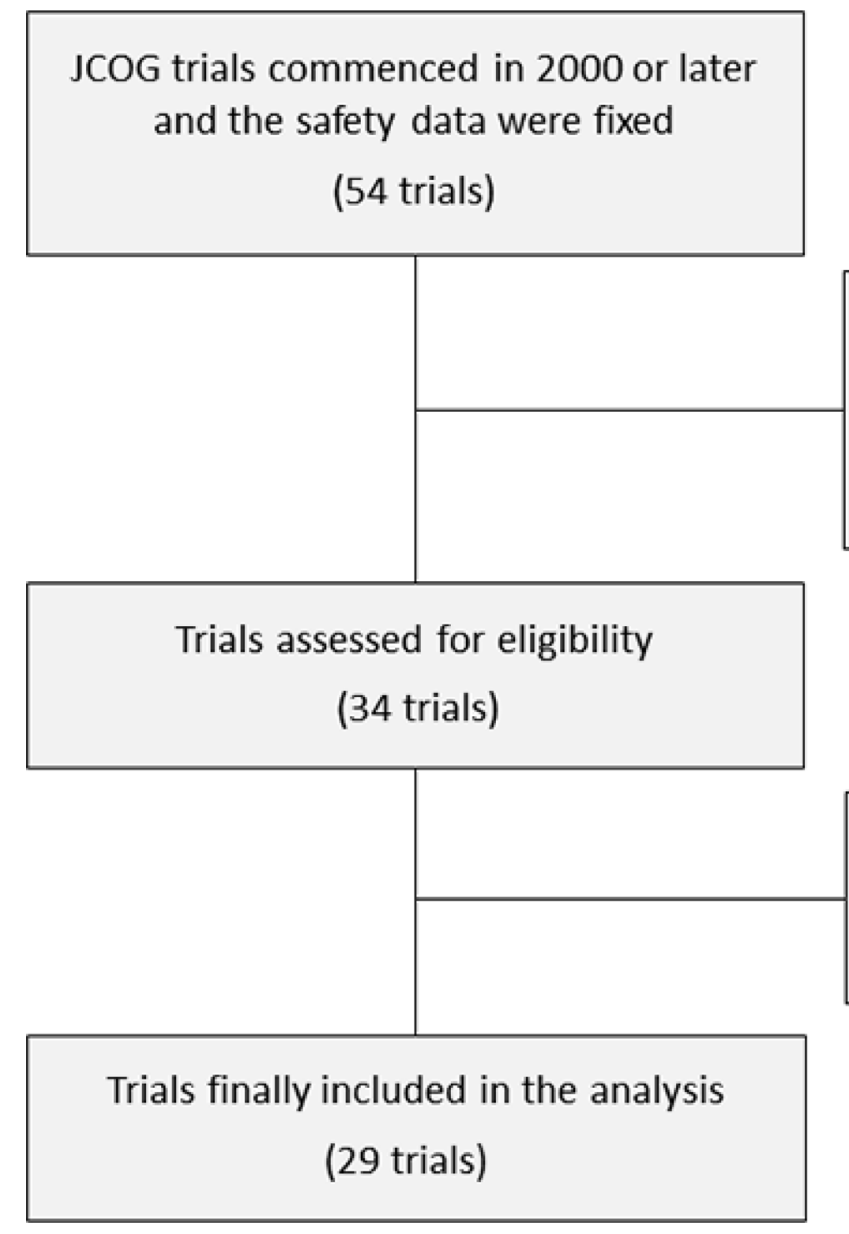

Figure 1: Selection process for the JCOG trials.

\section{Influence of underlying tumor type}

The incidence of grade 3/4 hyponatremia was highest among patients with small-cell lung cancer (19.9\%), followed by esophageal/head and neck cancer $(14.2 \%)$, non-small-cell lung cancer $(10.7 \%)$, ovarian, fallopian tube and peritoneal cancer $(9.1 \%)$, gastric cancer $(9.0 \%)$, cervical cancer $(5.7 \%)$, and bladder cancer $(5.4 \%)$. The differences among tumor types were statistically significant $(P<0.01)$. The incidence of grade 4 hyponatremia was relatively higher in small-cell lung cancer $(5.1 \%)$ than in the other cancers $(P<0.01)$.

\section{Influence of patients' age}

The incidences of grade 3/4 hyponatremia in elderly and nonelderly patients were $11.2 \%$ and $12.0 \%$, respectively. There was no significant difference between these subgroups $(P=0.77)$. The incidence of grade 4 hyponatremia showed a similar tendency to that of grade 3/4 hyponatremia $(P=0.30)$.

\section{Excluded}

No systemic chemotherapy Nonsolid tumors

Soft tissue sarcoma

\section{(20 trials)}

(16 trials) (3 trials) (1 trial)
Excluded

No sodium data Unpublished trial
(5 trials)

(3 trials) (2 trials) 
Table 1A: Characteristics of JCOG clinical trials included in this study (Platinum-based chemotherapy arms)

\begin{tabular}{|c|c|c|c|c|c|c|c|c|c|}
\hline $\begin{array}{l}\text { JCOG trial } \\
\text { No. }\end{array}$ & Phase & Malignancy & $\begin{array}{c}\text { No. of } \\
\text { patients }\end{array}$ & Regimen & $\begin{array}{c}\text { Platinum } \\
\text { administration } \\
\text { schedule }\end{array}$ & $\begin{array}{c}\text { Cisplatin } \\
\text { administration } \\
\text { method }\end{array}$ & $\begin{array}{c}\text { Cisplatin } \\
\text { dosage } \\
\text { (DI, } \mathbf{m g} / \mathbf{m}^{2} / \\
\text { week) }\end{array}$ & $\begin{array}{l}\text { Median age } \\
\text { (years) }\end{array}$ & Reference \\
\hline 0001 & II & Gastric & 55 & $\begin{array}{l}\text { Cisplatin and } \\
\text { irinotecan }\end{array}$ & $\begin{array}{c}80 \mathrm{mg} / \mathrm{m}^{2} \text { on day } 1 \\
\text { every } 4 \text { weeks for } 8 \text { or } \\
12 \text { weeks }\end{array}$ & bolus & $\begin{array}{l}\text { high } \\
(20)\end{array}$ & 63 & 12 \\
\hline 0102 & III & Cervical & 66 & $\begin{array}{l}\text { Cisplatin, } \\
\text { bleomycin, } \\
\text { mitomycin and } \\
\text { vincristine }\end{array}$ & $\begin{array}{c}14 \mathrm{mg} / \mathrm{m}^{2} \text { on days } 1-5 \\
\text { every } 3 \text { weeks for } 6 \\
\text { weeks }\end{array}$ & split & $\begin{array}{l}\text { high } \\
(23.3)\end{array}$ & 47 & 13 \\
\hline 0204 & rII & $\begin{array}{l}\text { Non-small-cell } \\
\text { lung }\end{array}$ & 40 & $\begin{array}{l}\text { Cisplatin and } \\
\text { docetaxel }\end{array}$ & $\begin{array}{c}80 \mathrm{mg} / \mathrm{m}^{2} \text { on day } 1 \\
\text { every } 4 \text { weeks for } 8 \\
\text { weeks }\end{array}$ & bolus & $\begin{array}{l}\text { high } \\
(20)\end{array}$ & 62.5 & 19 \\
\hline \multirow{2}{*}{0206} & \multirow{2}{*}{ FS } & \multirow{2}{*}{$\begin{array}{l}\text { Ovarian, fallopian } \\
\text { tube, peritoneal }\end{array}$} & 53 & $\begin{array}{l}\text { Carboplatin } \\
\text { and paclitaxel } \\
\text { (neoadjuvant) }\end{array}$ & $\begin{array}{l}\text { AUC } 6 \text { on day } 1 \text { every } \\
3 \text { weeks for } 12 \text { weeks }\end{array}$ & - & - & \multirow{2}{*}{55} & \multirow{2}{*}{21} \\
\hline & & & 46 & $\begin{array}{l}\text { Carboplatin } \\
\text { and paclitaxel } \\
\text { (adjuvant) }\end{array}$ & $\begin{array}{l}\text { AUC } 6 \text { on day } 1 \text { every } \\
3 \text { weeks for } 12 \text { weeks }\end{array}$ & - & - & & \\
\hline 0207 & III & $\begin{array}{l}\text { Non-small-cell } \\
\text { lung }\end{array}$ & 63 & $\begin{array}{l}\text { Cisplatin and } \\
\text { docetaxel }\end{array}$ & $\begin{array}{l}25 \mathrm{mg} / \mathrm{m}^{2} \text { on days } 1,8 \\
\text { and } 15 \text { every } 4 \text { weeks }\end{array}$ & split & $\begin{array}{l}\text { low } \\
(18.8)\end{array}$ & 76 & 22 \\
\hline 0209 & III & Bladder & 56 & $\begin{array}{l}\text { Cisplatin, } \\
\text { methotrexate, } \\
\text { vinblastine and } \\
\text { Adriamycin }\end{array}$ & $\begin{array}{c}70 \mathrm{mg} / \mathrm{m}^{2} \text { on day } 2 \\
\text { every } 4 \text { weeks for } 8 \\
\text { weeks }\end{array}$ & bolus & $\begin{array}{l}\text { low } \\
(17.5)\end{array}$ & 63 & 23 \\
\hline 0210 & II & Gastric & 49 & $\begin{array}{c}\text { Cisplatin and } \\
\text { S1 }\end{array}$ & $\begin{array}{c}60 \mathrm{mg} / \mathrm{m}^{2} \text { on day } 8 \\
\text { every } 4 \text { weeks for } 8 \\
\text { weeks }\end{array}$ & bolus & $\begin{array}{l}\text { low } \\
(15)\end{array}$ & 61 & 24 \\
\hline 0301 & III & $\begin{array}{l}\text { Non-small-cell } \\
\text { lung }\end{array}$ & 96 & Carboplatin & $\begin{array}{c}30 \mathrm{mg} / \mathrm{m}^{2} \text { on days } 1-5 \\
\text { weekly for } 4 \text { weeks }\end{array}$ & - & - & 77 & 25 \\
\hline \multirow[t]{2}{*}{0303} & \multirow[t]{2}{*}{ III } & \multirow[t]{2}{*}{ Esophageal } & 70 & $\begin{array}{l}\text { Cisplatin and } \\
\text { fluorouracil }\end{array}$ & $\begin{array}{c}70 \mathrm{mg} / \mathrm{m}^{2} \text { on day } 1 \\
\text { every } 4 \text { weeks for } 8 \\
\text { weeks }\end{array}$ & bolus & $\begin{array}{l}\text { low } \\
(17.5)\end{array}$ & 63 & \multirow[t]{2}{*}{26} \\
\hline & & & 70 & $\begin{array}{l}\text { Cisplatin and } \\
\text { fluorouracil }\end{array}$ & $\begin{array}{c}4 \mathrm{mg} / \mathrm{m}^{2} \text { on days } 1-5 \\
\text { weekly for } 6 \text { weeks }\end{array}$ & split & $\begin{array}{l}\text { high } \\
(20)\end{array}$ & 62 & \\
\hline 0402 & $\mathrm{I} / \mathrm{II}^{*}$ & $\begin{array}{l}\text { Non-small-cell } \\
\text { lung }\end{array}$ & 38 & $\begin{array}{l}\text { Cisplatin and } \\
\text { vinorelbine }\end{array}$ & $\begin{array}{c}80 \mathrm{mg} / \mathrm{m}^{2} \text { on day } 1 \\
\text { every } 3 \text { weeks for } 6 \\
\text { weeks }\end{array}$ & bolus & $\begin{array}{l}\text { high } \\
(26.7)\end{array}$ & 59.5 & 27 \\
\hline 0405 & II & Gastric & 52 & $\begin{array}{l}\text { Cisplatin and } \\
\text { S1 }\end{array}$ & $\begin{array}{c}60 \mathrm{mg} / \mathrm{m}^{2} \text { on day } 8 \\
\text { every } 4 \text { weeks for } 8 \text { or } \\
12 \text { weeks }\end{array}$ & bolus & $\begin{array}{l}\text { how } \\
(15)\end{array}$ & 63 & 28 \\
\hline 0502 & III & Esophageal & 165 & $\begin{array}{l}\text { Cisplatin and } \\
\text { fluorouracil }\end{array}$ & $\begin{array}{c}70 \mathrm{mg} / \mathrm{m}^{2} \text { on day } 1 \\
\text { every } 4 \text { weeks for } 8 \\
\text { weeks }\end{array}$ & bolus & $\begin{array}{l}\text { low } \\
(17.5)\end{array}$ & $\begin{array}{l}\mathrm{B} ; 69 \\
\mathrm{D} ; 65\end{array}$ & unpublished \\
\hline \multirow[t]{2}{*}{0505} & \multirow[t]{2}{*}{ III } & \multirow[t]{2}{*}{ Cervical } & 125 & $\begin{array}{l}\text { Cisplatin and } \\
\text { paclitaxel }\end{array}$ & $\begin{array}{c}50 \mathrm{mg} / \mathrm{m}^{2} \text { on day } 2 \\
\text { every } 3 \text { weeks for } 18 \\
\text { weeks }\end{array}$ & bolus & $\begin{array}{l}\text { low } \\
(16.7)\end{array}$ & 53 & \multirow[t]{2}{*}{31} \\
\hline & & & 126 & $\begin{array}{c}\text { Carboplatin } \\
\text { and paclitaxel }\end{array}$ & $\begin{array}{l}\text { AUC } 5 \text { on day } 1 \text { every } \\
3 \text { weeks for } 18 \text { weeks }\end{array}$ & - & - & 53 & \\
\hline 0508 & II & Esophageal & 96 & $\begin{array}{l}\text { Cisplatin and } \\
\text { fluorouracil }\end{array}$ & $\begin{array}{c}70 \mathrm{mg} / \mathrm{m}^{2} \text { on day } 1 \\
\text { every } 4 \text { weeks for } 8 \\
\text { weeks }\end{array}$ & bolus & $\begin{array}{l}\text { low } \\
(17.5)\end{array}$ & 63 & unpublished \\
\hline \multirow{2}{*}{0509} & \multirow{2}{*}{ III } & \multirow{2}{*}{ Small-cell lung } & 142 & $\begin{array}{l}\text { Cisplatin and } \\
\text { irinotecan }\end{array}$ & $\begin{array}{c}60 \mathrm{mg} / \mathrm{m}^{2} \text { on day } 1 \\
\text { every } 4 \text { weeks for } 16 \\
\text { weeks }\end{array}$ & bolus & $\begin{array}{l}\text { low } \\
(15)\end{array}$ & 63 & \multirow{2}{*}{33} \\
\hline & & & 140 & $\begin{array}{l}\text { Cisplatin and } \\
\text { amrubicin }\end{array}$ & $\begin{array}{c}60 \mathrm{mg} / \mathrm{m}^{2} \text { on day } 1 \\
\text { every } 3 \text { weeks for } 12 \\
\text { weeks }\end{array}$ & bolus & $\begin{array}{l}\text { high } \\
(20)\end{array}$ & 63 & \\
\hline
\end{tabular}




\begin{tabular}{|c|c|c|c|c|c|c|c|c|c|}
\hline & & & 137 & $\begin{array}{l}\text { Carboplatin } \\
\text { and paclitaxel } \\
\text { (adjuvant) }\end{array}$ & $\begin{array}{c}\text { AUC } 6 \text { on day } 1 \text { every } \\
3 \text { weeks for } 24 \text { weeks } \\
\text { (adjuvant) }\end{array}$ & - & - & 59 & \\
\hline 0602 & III & $\begin{array}{l}\text { Ovarian, fallopian } \\
\text { tube, peritoneal }\end{array}$ & 149 & $\begin{array}{c}\text { Carboplatin } \\
\text { and paclitaxel } \\
\text { (neoadjuvant } \\
\text { plus adjuvant) }\end{array}$ & $\begin{array}{c}\text { AUC } 6 \text { on day } 1 \text { every } \\
3 \text { weeks for } 12 \text { weeks } \\
\text { (neoadjuvant) plus } 12 \\
\text { weeks (adjuvant) }\end{array}$ & - & - & 60.5 & unpublished \\
\hline 0604 & $\mathrm{I} / \mathrm{II}^{*}$ & Esophageal & 38 & $\begin{array}{l}\text { Cisplatin and } \\
\text { S1 }\end{array}$ & $\begin{array}{c}75 \mathrm{mg} / \mathrm{m}^{2} \text { on day } 1 \\
\text { every } 4 \text { weeks for } 8 \\
\text { weeks }\end{array}$ & bolus & $\begin{array}{l}\text { low } \\
(18.8)\end{array}$ & 62 & 34 \\
\hline 0605 & III & Small-cell lung & 90 & $\begin{array}{l}\text { Cisplatin, } \\
\text { etoposide, and } \\
\text { irinotecan }\end{array}$ & $\begin{array}{c}25 \mathrm{mg} / \mathrm{m}^{2} \text { on days } 1,8 \\
\text { every } 2 \text { weeks for } 10 \\
\text { weeks }\end{array}$ & split & $\begin{array}{l}\text { high } \\
(25)\end{array}$ & 64 & 35 \\
\hline \multirow{2}{*}{0706} & \multirow{2}{*}{ II } & \multirow{2}{*}{ Head and neck } & 45 & $\begin{array}{c}\text { Cisplatin and } \\
\text { S1 }\end{array}$ & $\begin{array}{l}20 \mathrm{mg} / \mathrm{m}^{2} \text { on days } \\
8-11 \text { every } 5 \text { weeks } \\
\text { for } 10 \text { weeks }\end{array}$ & split & $\begin{array}{l}\text { low } \\
(16)\end{array}$ & 63 & \multirow{2}{*}{36} \\
\hline & & & 40 & $\begin{array}{l}\text { Cisplatin and } \\
\text { S1 }\end{array}$ & $\begin{array}{l}20 \mathrm{mg} / \mathrm{m}^{2} \text { on days } \\
8-11 \text { every } 4 \text { weeks } \\
\text { for } 8 \text { weeks }\end{array}$ & split & $\begin{array}{l}\text { high } \\
(20)\end{array}$ & & \\
\hline 0803 & III & $\begin{array}{l}\text { Non-small-cell } \\
\text { lung }\end{array}$ & 137 & $\begin{array}{l}\text { Cisplatin and } \\
\text { docetaxel }\end{array}$ & $\begin{array}{l}25 \mathrm{mg} / \mathrm{m}^{2} \text { on days } 1,8 \\
\text { and } 15 \text { every } 4 \text { weeks }\end{array}$ & split & $\begin{array}{l}\text { low } \\
(18.8)\end{array}$ & 76 & 37 \\
\hline 0807 & $\mathrm{I} / \mathrm{II}^{*}$ & Esophageal & 55 & $\begin{array}{l}\text { Cisplatin, } \\
\text { fluorouracil, } \\
\text { and docetaxel }\end{array}$ & $\begin{array}{c}80 \mathrm{mg} / \mathrm{m}^{2} \text { on day } 1 \\
\text { every } 4 \text { weeks }\end{array}$ & bolus & $\begin{array}{l}\text { high } \\
(20)\end{array}$ & 61 & 38 \\
\hline
\end{tabular}

Abbreviations: rII, randomized phase II; FS, feasibility study; AUC, area under the blood concentration time curve; I/II; phase I/II; DI, dose intensity. *Data from phase II were used in this study.

Table 1B: Characteristics of JCOG clinical trials included in this study (Nonplatinum-based chemotherapy arms)

\begin{tabular}{|c|c|c|c|c|c|c|}
\hline $\begin{array}{l}\text { JCOG trial } \\
\text { No. }\end{array}$ & Phase & Malignancy & No. of patients & Regimen & $\begin{array}{c}\text { Median age } \\
\text { (years) }\end{array}$ & Reference \\
\hline \multirow{2}{*}{0104} & \multirow{2}{*}{ III } & \multirow{2}{*}{ Non-small-cell lung } & 64 & Docetaxel & 62 & \multirow{2}{*}{14} \\
\hline & & & 65 & Docetaxel and gemcitabine & 60 & \\
\hline \multirow[b]{2}{*}{0106} & \multirow[b]{2}{*}{ III } & \multirow[b]{2}{*}{ Gastric } & 117 & Fluorouracil & 61 & \multirow[b]{2}{*}{15} \\
\hline & & & 116 & $\begin{array}{l}\text { Fluorouracil and } \\
\text { methotrexate }\end{array}$ & 59 & \\
\hline \multirow[t]{2}{*}{0111} & \multirow[t]{2}{*}{ III } & \multirow[t]{2}{*}{ Breast } & 7 & $\begin{array}{c}\text { Anthracycline (every } 3 \\
\text { weeks) }\end{array}$ & 59 & \multirow[t]{2}{*}{16} \\
\hline & & & 6 & Anthracycline (weekly) & 50 & \\
\hline 0204 & rII & Non-small-cell lung & 40 & Docetaxel & 66 & 19 \\
\hline \multirow{2}{*}{0205} & \multirow{2}{*}{ III } & \multirow{2}{*}{ Colorectal } & 542 & Fluorouracil and leucovorin & 61 & \multirow{2}{*}{20} \\
\hline & & & 536 & UFT and leucovorin & 61 & \\
\hline 0207 & III & Non-small-cell lung & 62 & Docetaxel & 76 & 22 \\
\hline \multirow[t]{2}{*}{0407} & \multirow[t]{2}{*}{ rII } & \multirow[t]{2}{*}{ Gastric } & 49 & $\begin{array}{l}\text { Fluorouracil with or without } \\
\text { methotrexate }\end{array}$ & 59 & \multirow[t]{2}{*}{29} \\
\hline & & & 51 & Paclitaxel & 64 & \\
\hline 0503 & II & Ovarian & 60 & irinotecan and etoposide & 58 & 30 \\
\hline 0506 & II & Pancreatic & 50 & Gemcitabine & 67.5 & 32 \\
\hline 0605 & III & Small-cell lung & 90 & Nogitecan & 64 & 35 \\
\hline 0803 & III & Non-small-cell lung & 137 & Docetaxel & 76 & 37 \\
\hline 0901 & II & Small-cell lung & 82 & Amrubicin & 66 & 39 \\
\hline
\end{tabular}

Abbreviations: rII, randomized phase II. 


\section{DISCUSSION}

We conducted a cross-sectional, descriptive epidemiological study using data from completed clinical trials undertaken by JCOG in order to investigate the incidence of platinum-induced hyponatremia and its associated potential risk factors. The study design provided the following advantages. (a) By using data from patients who were enrolled into clinical trials without major deficiency of organ function, most cases of hyponatremia with causes other than the administration of platinum agents could be excluded. (b) We could obtain an adequate sample size; moreover, hyponatremia related to various administration methods and patient backgrounds enabled us to investigate potential risk factors. (c) Because JCOG trials are managed under JCOG policies by means of periodic data submission and quality control of the collected data, we could use good-quality data without missing values. Based on these advantages, we believe that the results of this study are quite reliable.

Overall, grade $3 / 4$ hyponatremia developed in $11.9 \%$ of patients who received platinum agents. This proportion was significantly higher than that observed in patients who did not receive platinum agents (3.8\%), thereby supporting the hypothesis that platinum agents increase the risk of hyponatremia. However, among the studies that administered platinum agents, the incidence of platinum-induced hyponatremia varied greatly from $2.9 \%$ to $29.1 \%$. Therefore, it follows that treatment with platinum agents should be administered with care.

No treatment-related deaths due to hyponatremia (grade 5) were observed in any of the trials included in this study. In each of the trials included, early detection and appropriate treatment of hyponatremia must have been provided, and they were effective in preventing grade 5 hyponatremia.

In our study, we performed univariable analysis to determine possible risk factors. The results indicated that factors associated with a high incidence of hyponatremia included cisplatin usage, a cisplatin administration dosage of $\geq 20 \mathrm{mg} / \mathrm{m}^{2} /$ week, administration of threedrug combination regimen, administration of three-drug combination regimen with amrubicin or irinotecan, and small-cell lung cancer. In addition, because there were no differences in the incidence of hyponatremia associated with patient age or the platinum agent administration method, these factors may not be potential risk factors.

In terms of the type of platinum agent, the incidence of hyponatremia was significantly higher with cisplatin compared with carboplatin $(13.5 \%$ vs $7.6 \% ; P<0.01)$; this could be attributed to the difference in renal toxicity profiles of both drugs. Because carboplatin is excreted
Grade 3/4

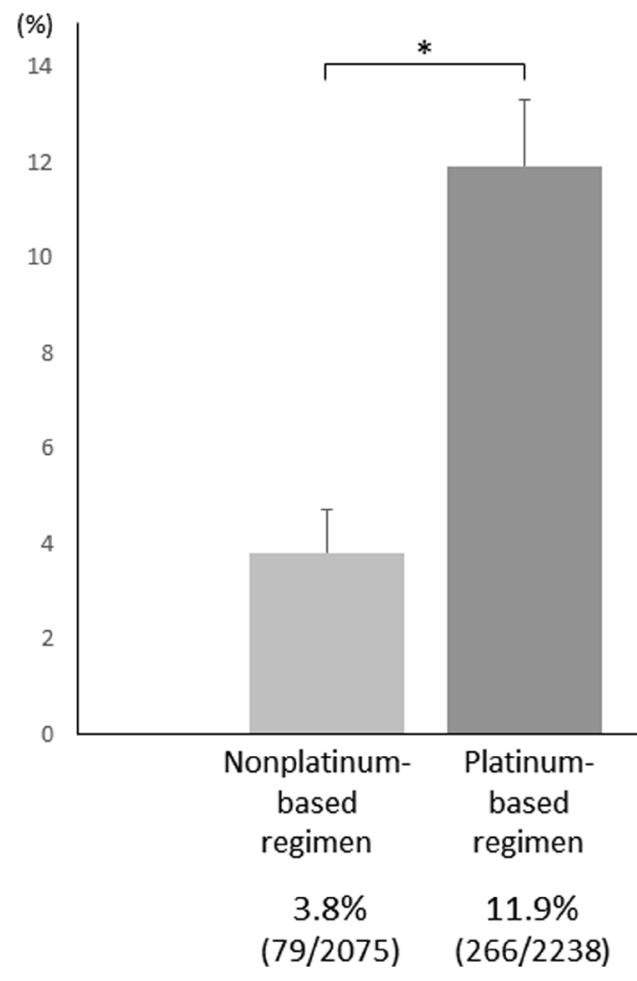

Grade 4 


\begin{tabular}{|c|c|c|c|c|c|c|c|c|c|c|}
\hline \multirow[b]{2}{*}{ Subgroup } & \multirow[b]{2}{*}{$\begin{array}{l}\text { No. of } \\
\text { treatment } \\
\text { arms }\end{array}$} & \multirow[b]{2}{*}{$\begin{array}{c}\text { No. of } \\
\text { patients }\end{array}$} & \multicolumn{4}{|c|}{ Grade 3/4 } & \multicolumn{4}{|c|}{ Grade 4} \\
\hline & & & $\begin{array}{l}\text { No. of } \\
\text { events }\end{array}$ & $\begin{array}{l}\text { Incidence } \\
(\%)\end{array}$ & $95 \%$ CI & $P$ value & $\begin{array}{l}\text { No. of } \\
\text { events }\end{array}$ & $\begin{array}{l}\text { Incidence } \\
\quad(\%)\end{array}$ & $95 \%$ CI & $P$ value \\
\hline \multicolumn{11}{|l|}{ Type of platinum agent } \\
\hline Carboplatin & 6 & 607 & 46 & 7.6 & $5.6-10.0$ & $<0.01$ & 2 & 0.3 & $0.0-1.2$ & $<0.01$ \\
\hline Cisplatin & 21 & 1631 & 220 & 13.5 & $11.9-15.2$ & & 31 & 1.9 & $1.3-2.7$ & \\
\hline \multicolumn{11}{|l|}{ Administration methods } \\
\hline Split & 8 & 606 & 69 & 11.4 & $9.0-14.2$ & 0.71 & 6 & 1.0 & $0.4-2.1$ & 0.32 \\
\hline Bolus & 19 & 1632 & 197 & 12.1 & $10.5-13.8$ & & 27 & 1.7 & $1.1-2.4$ & \\
\hline \multicolumn{11}{|l|}{$\begin{array}{l}\text { Weekly equivalent cisplatin } \\
\text { dose, } \mathrm{mg} / \mathrm{m}^{2}\end{array}$} \\
\hline$<20$ & 12 & 1037 & 127 & 12.2 & $10.3-14.4$ & 0.06 & 14 & 1.4 & $0.7-2.3$ & 0.04 \\
\hline$\geq 20$ & 9 & 594 & 93 & 15.7 & $12.8-18.8$ & & 17 & 2.9 & $1.7-4.5$ & \\
\hline \multicolumn{11}{|l|}{$\begin{array}{l}\text { Number of concomitant } \\
\text { medications }\end{array}$} \\
\hline 0 (Platinum alone) & 1 & 96 & 7 & 7.3 & $3.0-14.5$ & $<0.01$ & 0 & 0.0 & $0.0-3.8$ & 0.03 \\
\hline $\begin{array}{l}1 \text { (two-drug combination } \\
\text { regimen) }\end{array}$ & 22 & 1875 & 222 & 11.8 & $10.4-13.4$ & & 27 & 1.4 & $1.0-2.1$ & \\
\hline $\begin{array}{l}2 \text { (three-drug combination } \\
\text { regimen) }\end{array}$ & 2 & 145 & 31 & 21.4 & $15.0-29.0$ & & 6 & 4.1 & $1.5-8.8$ & \\
\hline $\begin{array}{l}3 \text { (four-drug combination } \\
\text { regimen) }\end{array}$ & 2 & 122 & 6 & 4.9 & $1.8-10.4$ & & 0 & 0.0 & $0.0-3.0$ & \\
\hline \multicolumn{11}{|l|}{$\begin{array}{l}\text { Types of concomitant } \\
\text { medications }\end{array}$} \\
\hline Vinorelbine & 1 & 38 & 1 & 2.6 & $0.1-13.8$ & $<0.01$ & 0 & 0.0 & $0.0-9.3$ & $<0.01$ \\
\hline Taxane & 9 & 875 & 82 & 9.4 & $7.5-11.5$ & & 6 & 0.7 & $0.3-1.5$ & \\
\hline Fluorouracil & 9 & 625 & 72 & 11.8 & $9.1-14.3$ & & 4 & 0.6 & $0.2-1.6$ & \\
\hline Irinotecan & 2 & 197 & 36 & 18.3 & $13.1-24.4$ & & 8 & 4.1 & $1.8-7.8$ & \\
\hline Amrubicin & 1 & 140 & 31 & 22.1 & $15.6-29.9$ & & 9 & 6.4 & $3.0-11.9$ & \\
\hline \multicolumn{11}{|l|}{ Tumor type } \\
\hline Bladder & 1 & 56 & 3 & 5.4 & $1.1-14.9$ & $<0.01$ & 0 & 0.0 & $0.0-6.4$ & $<0.01$ \\
\hline Cervical & 3 & 317 & 18 & 5.7 & $3.4-8.8$ & & 2 & 0.6 & $0.1-2.3$ & \\
\hline Gastric & 3 & 156 & 14 & 9.0 & $5.0-14.6$ & & 1 & 0.6 & $0.0-3.5$ & \\
\hline $\begin{array}{l}\text { Ovarian, fallopian tube } \\
\text { and peritoneal }\end{array}$ & 4 & 385 & 35 & 9.1 & $6.4-12.4$ & & 2 & 0.5 & $0.1-1.9$ & \\
\hline Non-small-cell lung & 5 & 373 & 40 & 10.7 & $7.8-14.3$ & & 2 & 0.5 & $0.1-1.9$ & \\
\hline Esophageal/head and neck & 8 & 579 & 82 & 14.2 & $11.4-17.3$ & & 7 & 1.2 & $0.5-2.5$ & \\
\hline Small-cell lung & 3 & 372 & 74 & 19.9 & $16.0-24.3$ & & 19 & 5.1 & $3.1-7.9$ & \\
\hline \multicolumn{11}{|l|}{ Median age } \\
\hline$\geq 70$ years old & 3 & 295 & 33 & 11.2 & $10.6-13.5$ & 0.77 & 2 & 0.7 & $0.1-2.4$ & 0.30 \\
\hline$<70$ years old & 24 & 1943 & 233 & 12.0 & $7.8-15.4$ & & 31 & 1.6 & $1.1-2.3$ & \\
\hline
\end{tabular}

Abbreviations: CI, confidence interval.

via filtration from the renal glomerulus alone, with no involvement of renal tubules, it rarely causes tubular disorders. In contrast, after being excreted via filtration from the renal glomerulus, cisplatin is reabsorbed through the proximal tubule and transported into epithelial cells through the basal membrane from blood vessels around the interstitial tissue; therefore, cisplatin administration may cause tubular disorders [10, 40, 41]. Thus, the difference in the incidence of hyponatremia observed in this study might be attributed to differences in renal toxicity (tubular disorders). To avoid such renal toxicity, mass infusion or administration of diuretics is often provided when patients undergo chemotherapy with platinum agents. Nevertheless, such mass infusion or diuretics themselves can cause hyponatremia $[4,8,9]$. However, the results of our study did not reveal any significant differences in the incidence of hyponatremia between the bolus administration group that received mass infusion along 
with diuretics and the split administration group where these were not administered together $(12.1 \%$ vs $11.4 \% ; P=$ $0.71)$; therefore, such factors seem to have limited effects. In addition, anorexia often occurs with administration of platinum agents, and it can cause hyponatremia. However, findings from the trials included that reported anorexia demonstrated no significant correlation between grade 3/4 anorexia and grade 3/4 hyponatremia using Pearson's correlation analysis ( $P=0.46$; data not shown), suggesting that anorexia had a limited influence on hyponatremia. Overall, and as previously reported, the primary pathophysiology of platinum-induced hyponatremia may be renal salt-wasting syndrome and SIADH (syndrome of inappropriate antidiuretic hormone secretion).

Regarding the association between the number of concomitant medications and hyponatremia, the incidence of hyponatremia increased as the number of concomitant medications increased from the monotherapy group to the three-drug combination regimen group. However, unexpectedly, the lowest incidence of hyponatremia was observed with the four-drug combination regimen. Trials utilizing the four-drug combination regimen include the JCOG0102 trial [13], targeting cervical cancer, and the JCOG0209 trial [23], targeting bladder cancer; the JCOG0102 trial involved administration of cisplatin at $14 \mathrm{mg} / \mathrm{m}^{2}$ on days $1-5$, every 3 weeks for 6 weeks, and the JCOG0209 trial administered $70 \mathrm{mg} / \mathrm{m}^{2}$ on day 2, every 4 weeks for 8 weeks, indicating that dose intensity was not particularly low and there were no special conditions. The reason for the lowest incidence with the four-drug combination regimens remains unclear.
On investigating concomitant medications, our results indicated a specifically high incidence of hyponatremia for amrubicin and, by underlying tumor type, small-cell lung cancer. However, because amrubicin was only administrated in cases of small-cell lung cancer among the included trials in this study, we could not elucidate which of these two was the stronger influencing factor. The incidence of hyponatremia was $15.9 \%$ (13/82) in a trial where amrubicin was administered alone (JCOG0901) [39], which is extremely high compared with that of other trials in the nonplatinum-based chemotherapy group $(3.3 \% ; 66 / 1993)$. This might suggest that amrubicin itself has a marked effect on hyponatremia.

The primary limitation of this study was the fact that it was a descriptive epidemiological study that did not use individual raw data. Therefore, we were unable to analyze factors, such as the timing of hyponatremia onset, detailed profiles of patients in whom hyponatremia occurred, and any possible gender differences, which warrants an investigation of individual raw data. However, no treatment-related deaths or severe aftereffects due to hyponatremia, such as central pontine myelinolysis, were observed in any of the trials included in our study. Consequently, because our findings can be considered sufficiently valuable, as they demonstrate incidence and potential risk factors of platinum-induced hyponatremia, we did not pursue a more detailed investigation using individual raw data.

In conclusion, this study is the first report to demonstrate the actual incidence of platinum-induced hyponatremia and its associated potential risk factors

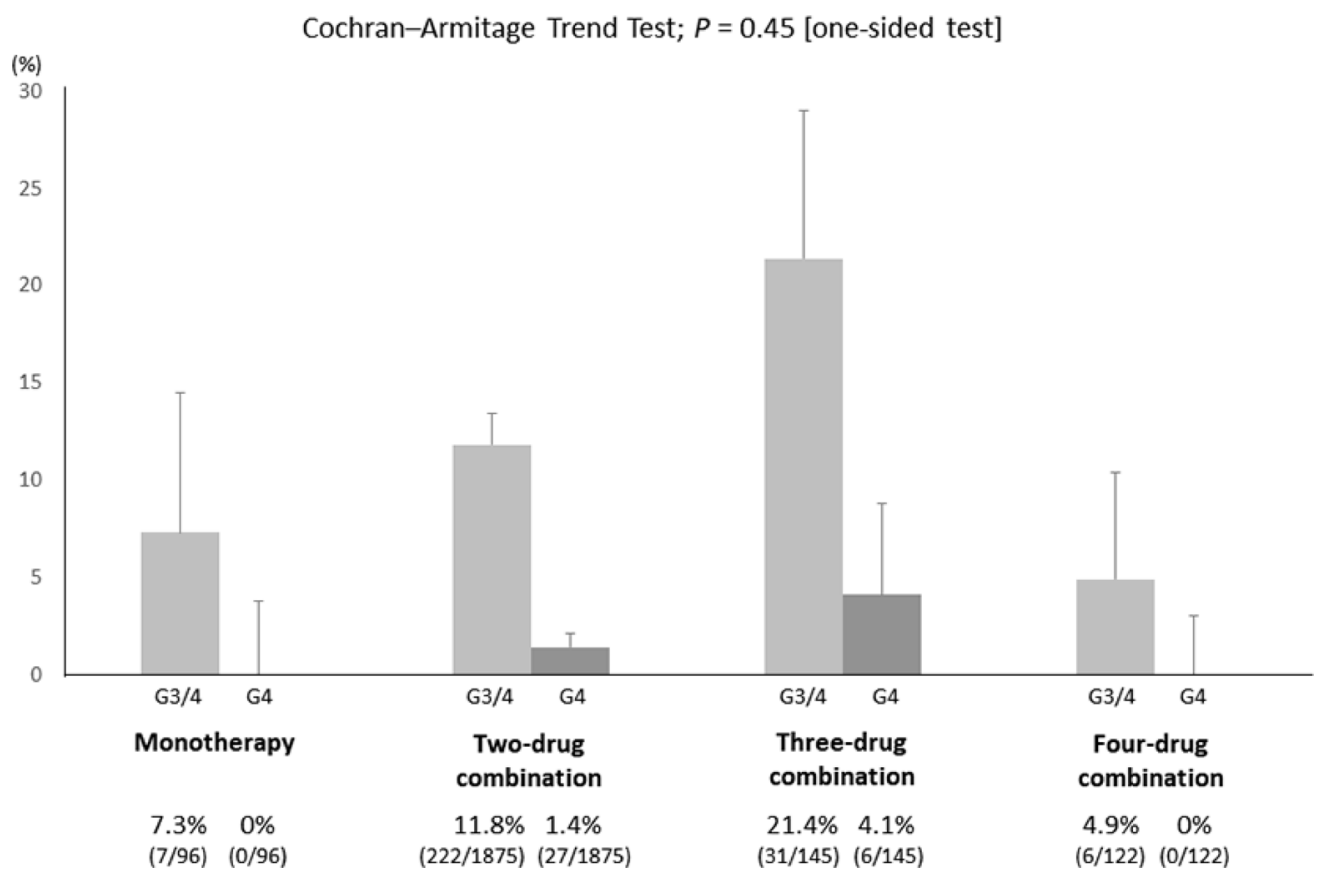

Figure 3: Incidence of platinum-induced hyponatremia in patients who received a platinum agent in monotherapy, two-drug, three-drug, and four-drug combination regimens. 
using a large-scale sample size and highly reliable data. These results will be useful for early detection and appropriate treatment of platinum-induced hyponatremia. Careful monitoring of serum sodium level is needed when platinum is administered. We anticipate that these results will be used to not only improve the safety of platinumbased chemotherapy, but also as a useful reference for future medical research.

\section{MATERIALS AND METHODS}

\section{Study selection}

JCOG clinical trials that met the following criteria were included in this analysis: (1) the study protocol had been approved by the protocol review committee of JCOG after January 2000; (2) patient enrolments had finished before January 2014; (3) phase II or III trials of patients with solid cancer who had received systemic chemotherapy; and (4) data on hyponatremia were available. Data from unpublished trials were excluded if the agreement to use the data was withheld by the representative of each trial.

\section{Data source}

Data about the incidence of grade 3/4 hyponatremia used for our analysis were evaluated using the National Cancer Institute-Common Toxicity Criteria (NCI-CTC) v. 2.0 or Common Terminology Criteria for Adverse Events (CTCAE) v. 3.0 or v. 4.0 as described in the most recent reports of included trials, the data of which are stored in the JCOG Data Center. In NCI-CTC v. 2.0 or CTCAE v. 3.0 and v. 4.0, grade 3 hyponatremia is defined as serum sodium concentration $120-<130 \mathrm{mmol} / \mathrm{L}$ and grade 4 as $<120 \mathrm{mmol} / \mathrm{L}$. In addition, from information provided in the trial reports, we confirmed the presence or absence of grade 5 hyponatremia, defined as a fatality, and any serious aftereffects related to hyponatremia. Data of individual patients stored in the database were not used in this study.

\section{Statistical analysis}

The incidence of grade 3/4 hyponatremia was compared between a platinum-based chemotherapy arm and nonplatinum-based chemotherapy arm. To explore the risk factors of platinum-induced hyponatremia, included trials of patients receiving platinum-based chemotherapy were divided into more than one subgroup according to the following criteria: type of platinum agent (cisplatin vs carboplatin), administration method of the platinum agent (bolus vs split), cisplatin dosage $\left(<20 \mathrm{mg} / \mathrm{m}^{2} /\right.$ week vs $\geq 20 \mathrm{mg} / \mathrm{m}^{2} /$ week), number of concomitant medications (platinum monotherapy alone, two-, three-, or four-drug combination regimens), type of concomitant medications (vinorelbine, taxane, fluorouracil, irinotecan, or amrubicin) in a two-drug combination regimen, underlying tumor type (bladder, cervical, gastric, ovarian, fallopian tube and peritoneal, non-small-cell lung, esophageal/head and neck, or small-cell lung), and patients' median age ( $<70$ years old vs $\geq 70$ years old). With regards to cisplatin, because various dosages and schedules were studied in the included trials, an equivalent weekly dosage was calculated to standardize cisplatin dosage (e.g., patients receiving $80 \mathrm{mg} / \mathrm{m}^{2}$ once every 4 weeks or $20 \mathrm{mg} / \mathrm{m}^{2}$ once per week would both be in the $20 \mathrm{mg} / \mathrm{m}^{2} /$ week subgroup). With regard to patients' median age, because three of 27 trials were studied with elderly patients, median age was categorized as a nonelderly subgroup ( $<70$ years old) or elderly subgroup ( $\geq 70$ years old). The incidence of grade $3 / 4$ hyponatremia along with $95 \%$ confidence interval (CI) by Clopper and Pearson method was calculated for each subgroup. Fisher's exact test was used to compare the incidence of hyponatremia among subgroups. A two-sided $P$ value $<0.05$ was considered statistically significant. Because all evaluations in this study are considered to be exploratory data analyses, multiplicity adjustment was not applied. Statistical analysis was performed using SAS software (v. 9.2+; SAS Institute, Cary, NC, USA).

\section{Author contributions}

Conception and design: All authors; Collection and assembly of data: Yasumasa Ezoe, Junki Mizusawa, Hiroshi Katayama, Kozo Kataoka; Data analysis and interpretation: Yasumasa Ezoe, Junki Mizusawa, Hiroshi Katayama, Manabu Muto; Manuscript writing: All authors; Final approval of manuscript: All authors; Accountable for all aspects of the work: All authors.

\section{ACKNOWLEDGMENTS}

We are grateful to the group chair of each study group in JCOG: Dr. Yuichiro Ohe (Lung Cancer Study Group), Dr. Hisao Asamura (Lung Cancer Surgical Study Group), Dr. Mitsuru Sasako (Stomach Cancer Study Group), Dr. Yuko Kitagawa (Japan Esophageal Oncology Group), Dr. Hiroji Iwata (Breast Cancer Study Group), Dr. Nobuo Yaegashi (Gynecologic Cancer Study Group), Dr. Yasuhiro Shimada (Colorectal Cancer Study Group), Dr. Yoshiyuki Kakei (Urologic Oncology Study Group), Dr. Junji Furuse (Hepatobiliary and Pancreatic Oncology Group), Dr. Masato Fujii (Head and Neck Cancer Study Group) and Dr. Kensei Tobinai (JCOG Chair), for the permission to use their data, and to the members of the JCOG Data Center for the proofreading of this manuscript.

\section{CONFLICTS OF INTEREST}

The authors declare no conflicts of interest. 


\section{FUNDING}

The work was supported in part by The National Cancer Center Research and Development Funds [26-A-4, 29-A-3].

\section{REFERENCES}

1. Abu Zeinah GF, Al-Kindi SG, Hassan AA, Allam A. Hyponatraemia in cancer: association with type of cancer and mortality. Eur J Cancer Care (Engl). 2015; 24:224-31. https://doi.org/10.1111/ecc.12187.

2. Berardi R, Rinaldi S, Caramanti M, Grohe C, Santoni M, Morgese F, Torniai M, Savini A, Fiordoliva I, Cascinu S. Hyponatremia in cancer patients: Time for a new approach. Crit Rev Oncol Hematol. 2016; 102:15-25. https://doi. org/10.1016/j.critrevonc.2016.03.010.

3. Castillo JJ, Glezerman IG, Boklage SH, Chiodo J 3rd, Tidwell BA, Lamerato LE, Schulman KL. The occurrence of hyponatremia and its importance as a prognostic factor in a cross-section of cancer patients. BMC Cancer. 2016; 16: 564. https://doi.org/10.1186/s12885-016-2610-9.

4. Adrogue HJ, Madias NE. Hyponatremia. N Engl J Med. 2000; 342:1581-9. https://doi.org/10.1056/NEJM200005253422107.

5. Liamis G, Milionis H, Elisaf M. A review of drug-induced hyponatremia. Am J Kidney Dis. 2008; 52:144-53. https:// doi.org/10.1053/j.ajkd.2008.03.004.

6. Anderson RJ, Chung HM, Kluge R, Schrier RW. Hyponatremia: a prospective analysis of its epidemiology and the pathogenetic role of vasopressin. Ann Intern Med. $1985 ; 102: 164-8$.

7. Castillo JJ, Vincent M, Justice E. Diagnosis and management of hyponatremia in cancer patients. Oncologist. 2012; 17:756-65. https://doi.org/10.1634/ theoncologist.2011-0400.

8. Raftopoulos H. Diagnosis and management of hyponatremia in cancer patients. Support Care Cancer. 2007; 15:1341-7. https://doi.org/10.1007/s00520-007-0309-9.

9. Berghmans T, Paesmans M, Body JJ. A prospective study on hyponatraemia in medical cancer patients: epidemiology, aetiology and differential diagnosis. Support Care Cancer. 2000; 8:192-7.

10. Berghmans T. Hyponatremia related to medical anticancer treatment. Support Care Cancer. 1996; 4:341-50.

11. Vanhees SL, Paridaens R, Vansteenkiste JF. Syndrome of inappropriate antidiuretic hormone associated with chemotherapy-induced tumour lysis in small-cell lung cancer: case report and literature review. Ann Oncol. 2000; 11:1061-5.

12. Yoshikawa T, Sasako M, Yamamoto S, Sano T, Imamura H, Fujitani K, Oshita H, Ito S, Kawashima Y, Fukushima N. Phase II study of neoadjuvant chemotherapy and extended surgery for locally advanced gastric cancer. Br J Surg. 2009; 96:1015-22. https://doi.org/10.1002/bjs.6665.

13. Katsumata N, Yoshikawa H, Kobayashi H, Saito T, Kuzuya K, Nakanishi T, Yasugi T, Yaegashi N, Yokota H, Kodama S, Mizunoe T, Hiura M, Kasamatsu T, et al. Phase III randomised controlled trial of neoadjuvant chemotherapy plus radical surgery vs radical surgery alone for stages IB2, IIA2, and IIB cervical cancer: a Japan Clinical Oncology Group trial (JCOG 0102). Br J Cancer. 2013; 108:1957-63. https://doi.org/10.1038/bjc.2013.179.

14. Takeda K, Negoro S, Tamura T, Nishiwaki Y, Kudoh S, Yokota S, Matsui K, Semba H, Nakagawa K, Takada Y, Ando M, Shibata T, Saijo N. Phase III trial of docetaxel plus gemcitabine versus docetaxel in second-line treatment for non-small-cell lung cancer: results of a Japan Clinical Oncology Group trial (JCOG0104). Ann Oncol. 2009; 20:835-41. https://doi.org/10.1093/annonc/mdn705.

15. Shirao K, Boku N, Yamada Y, Yamaguchi K, Doi T, Goto M, Nasu J, Denda T, Hamamoto Y, Takashima A, Fukuda H, Ohtsu A, Gastrointestinal Oncology Study Group of the Japan Clinical Oncology G. Randomized Phase III study of 5-fluorouracil continuous infusion vs. sequential methotrexate and 5-fluorouracil therapy in far advanced gastric cancer with peritoneal metastasis (JCOG0106). Jpn J Clin Oncol. 2013; 43:972-80. https://doi.org/10.1093/jjco/hyt114.

16. Iwata $H$. The transition of breast cancer treatment and Japan Clinical Oncology Group research over two decades. Jpn J Clin Oncol. 2012; 42:14-20. https://doi.org/10.1093/jjco/ hyr161.

17. Chou T, Tobinai K, Uike N, Asakawa T, Saito I, Fukuda H, Mizoroki F, Ando K, Iida S, Ueda R, Tsukasaki K, Hotta T, Lymphoma Study Group of Japan Clinical Oncology Group J. Melphalan-prednisolone and vincristinedoxorubicin-dexamethasone chemotherapy followed by prednisolone/interferon maintenance therapy for multiple myeloma: Japan Clinical Oncology Group Study, JCOG0112. Jpn J Clin Oncol. 2011; 41:586-9. https://doi. org/10.1093/jjco/hyq245.

18. Watanabe $\mathrm{T}$, Tobinai $\mathrm{K}$, Shibata $\mathrm{T}$, Tsukasaki $\mathrm{K}$, Morishima Y, Maseki N, Kinoshita T, Suzuki T, Yamaguchi M, Ando K, Ogura M, Taniwaki M, Uike N, et al. Phase II/III study of R-CHOP-21 versus R-CHOP-14 for untreated indolent B-cell non-Hodgkin's lymphoma: JCOG 0203 trial. J Clin Oncol. 2011; 29:3990-8. https:// doi.org/10.1200/JCO.2011.34.8508.

19. Kunitoh H, Kato H, Tsuboi M, Asamura H, Tada H, Nagai K, Mitsudomi T, Koike T, Nakagawa K, Ichinose Y, Okada M, Shibata T, Saijo N, et al. A randomised phase II trial of preoperative chemotherapy of cisplatin-docetaxel or docetaxel alone for clinical stage IB/II non-small-cell lung cancer results of a Japan Clinical Oncology Group trial (JCOG 0204). Br J Cancer. 2008; 99:852-7. https://doi. org/10.1038/sj.bjc.6604613. 
20. Shimada Y, Hamaguchi T, Mizusawa J, Saito N, Kanemitsu Y, Takiguchi N, Ohue M, Kato T, Takii Y, Sato T, Tomita N, Yamaguchi S, Akaike M, et al. Randomised phase III trial of adjuvant chemotherapy with oral uracil and tegafur plus leucovorin versus intravenous fluorouracil and levofolinate in patients with stage III colorectal cancer who have undergone Japanese D2/D3 lymph node dissection: final results of JCOG0205. Eur J Cancer. 2014; 50:2231-40. https://doi.org/10.1016/j.ejca.2014.05.025.

21. Onda T, Kobayashi H, Nakanishi T, Hatae M, Iwasaka T, Konishi I, Shibata T, Fukuda H, Kamura T, Yoshikawa H. Feasibility study of neoadjuvant chemotherapy followed by interval debulking surgery for stage III/IV ovarian, tubal, and peritoneal cancers: Japan Clinical Oncology Group Study JCOG0206. Gynecol Oncol. 2009; 113:57-62. https://doi.org/10.1016/j.ygyno.2008.12.027.

22. Tsukada H, Yokoyama A, Goto K, Shinkai T, Harada M, Ando M, Shibata T, Ohe Y, Tamura T, Saijo N, Lung Cancer Study Group of the Japan Clinical Oncology G. Randomized controlled trial comparing docetaxel-cisplatin combination with weekly docetaxel alone in elderly patients with advanced non-small-cell lung cancer: Japan Clinical Oncology Group (JCOG) 0207dagger. Jpn J Clin Oncol. 2015; 45:88-95. https://doi.org/10.1093/jjco/hyu176.

23. Kitamura $H$, Tsukamoto $T$, Shibata $T$, Masumori N, Fujimoto H, Hirao Y, Fujimoto K, Kitamura Y, Tomita Y, Tobisu K, Niwakawa M, Naito S, Eto M, et al. Randomised phase III study of neoadjuvant chemotherapy with methotrexate, doxorubicin, vinblastine and cisplatin followed by radical cystectomy compared with radical cystectomy alone for muscle-invasive bladder cancer: Japan Clinical Oncology Group Study JCOG0209. Ann Oncol. 2014; 25:1192-8. https://doi.org/10.1093/annonc/mdu126.

24. Iwasaki Y, Sasako M, Yamamoto S, Nakamura K, Sano T, Katai H, Tsujinaka T, Nashimoto A, Fukushima N, Tsuburaya A, Gastric Cancer Surgical Study Group of Japan Clinical Oncology G. Phase II study of preoperative chemotherapy with S-1 and cisplatin followed by gastrectomy for clinically resectable type 4 and large type 3 gastric cancers (JCOG0210). J Surg Oncol. 2013; 107:741-5. https://doi.org/10.1002/jso.23301.

25. Atagi S, Kawahara M, Yokoyama A, Okamoto H, Yamamoto N, Ohe Y, Sawa T, Ishikura S, Shibata T, Fukuda H, Saijo N, Tamura T, Japan Clinical Oncology Group Lung Cancer Study G. Thoracic radiotherapy with or without daily low-dose carboplatin in elderly patients with non-small-cell lung cancer: a randomised, controlled, phase 3 trial by the Japan Clinical Oncology Group (JCOG0301). Lancet Oncol. 2012; 13:671-8. https://doi.org/10.1016/ S1470-2045(12)70139-0.

26. Shinoda M, Ando N, Kato K, Ishikura S, Kato H, Tsubosa Y, Minashi K, Okabe H, Kimura Y, Kawano T, Kosugi S, Toh Y, Nakamura K, et al. Randomized study of low-dose versus standard-dose chemoradiotherapy for unresectable esophageal squamous cell carcinoma (JCOG0303). Cancer Sci. 2015; 106:407-12. https://doi.org/10.1111/cas.12622.

27. Niho S, Ohe $\mathrm{Y}$, Ishikura S, Atagi S, Yokoyama A, Ichinose Y, Okamoto H, Takeda K, Shibata T, Tamura T, Saijo N, Fukuoka M. Induction chemotherapy followed by gefitinib and concurrent thoracic radiotherapy for unresectable locally advanced adenocarcinoma of the lung: a multicenter feasibility study (JCOG 0402). Ann Oncol. 2012; 23:2253-8. https://doi.org/10.1093/annonc/mds012.

28. Tsuburaya A, Mizusawa J, Tanaka Y, Fukushima N, Nashimoto A, Sasako M, Stomach Cancer Study Group of the Japan Clinical Oncology G. Neoadjuvant chemotherapy with S-1 and cisplatin followed by D2 gastrectomy with para-aortic lymph node dissection for gastric cancer with extensive lymph node metastasis. Br J Surg. 2014; 101:65360. https://doi.org/10.1002/bjs.9484.

29. Nishina T, Boku N, Gotoh M, Shimada Y, Hamamoto Y, Yasui H, Yamaguchi K, Kawai H, Nakayama N, Amagai K, Mizusawa J, Nakamura K, Shirao K, et al. Randomized phase II study of second-line chemotherapy with the best available 5-fluorouracil regimen versus weekly administration of paclitaxel in far advanced gastric cancer with severe peritoneal metastases refractory to 5-fluorouracil-containing regimens (JCOG0407). Gastric Cancer. 2015. https://doi.org/10.1007/s10120-015-0542-8.

30. Matsumoto K, Katsumata N, Shibata T, Satoh T, Saitou M, Yunokawa M, Takano T, Nakamura K, Kamura T, Konishi I. Phase II trial of oral etoposide plus intravenous irinotecan in patients with platinum-resistant and taxane-pretreated ovarian cancer (JCOG0503). Gynecol Oncol. 2015; 136:218-23. https://doi.org/10.1016/j.ygyno.2014.10.026.

31. Kitagawa R, Katsumata N, Shibata T, Kamura T, Kasamatsu T, Nakanishi T, Nishimura S, Ushijima K, Takano M, Satoh T, Yoshikawa H. Paclitaxel Plus Carboplatin Versus Paclitaxel Plus Cisplatin in Metastatic or Recurrent Cervical Cancer: The Open-Label Randomized Phase III Trial JCOG0505. J Clin Oncol. 2015; 33:2129-35. https://doi.org/10.1200/JCO.2014.58.4391.

32. Ishii H, Furuse J, Boku N, Okusaka T, Ikeda M, Ohkawa S, Fukutomi A, Hamamoto Y, Nakamura K, Fukuda H, Group JGOS. Phase II study of gemcitabine chemotherapy alone for locally advanced pancreatic carcinoma: JCOG0506. Jpn J Clin Oncol. 2010; 40:573-9. https://doi.org/10.1093/jjco/hyq011.

33. Satouchi M, Kotani Y, Shibata T, Ando M, Nakagawa K, Yamamoto N, Ichinose Y, Ohe Y, Nishio M, Hida T, Takeda K, Kimura T, Minato K, et al. Phase III study comparing amrubicin plus cisplatin with irinotecan plus cisplatin in the treatment of extensive-disease small-cell lung cancer: JCOG 0509. J Clin Oncol. 2014; 32:1262-8. https://doi.org/10.1200/JCO.2013.53.5153.

34. Tahara M, Fuse N, Mizusawa J, Sato A, Nihei K, Kanato K, Kato K, Yamazaki K, Muro K, Takaishi H, Boku N, Ohtsu A. Phase I/II trial of chemoradiotherapy with concurrent S-1 and cisplatin for clinical stage II/III 
esophageal carcinoma (JCOG 0604). Cancer Sci. 2015; 106:1414-20. https://doi.org/10.1111/cas.12764.

35. Goto K, Ohe $\mathrm{Y}$, Shibata $\mathrm{T}$, Seto $\mathrm{T}$, Takahashi $\mathrm{T}$, Nakagawa K, Tanaka H, Takeda K, Nishio M, Mori K, Satouchi M, Hida T, Yoshimura N, et al. Combined chemotherapy with cisplatin, etoposide, and irinotecan versus topotecan alone as second-line treatment for patients with sensitive relapsed small-cell lung cancer (JCOG0605): a multicentre, open-label, randomised phase 3 trial. Lancet Oncol. 2016; 17:1147-57. https://doi.org/10.1016/ S1470-2045(16)30104-8.

36. Tahara M, Kiyota N, Mizusawa J, Nakamura K, Hayashi R, Akimoto T, Hasegawa Y, Iwae S, Monden N, Matsuura K, Fujii H, Onozawa Y, Homma A, et al. Phase II trial of chemoradiotherapy with S-1 plus cisplatin for unresectable locally advanced head and neck cancer (JCOG0706). Cancer Sci. 2015; 106:726-33. https://doi.org/10.1111/cas.12657.

37. Abe T, Takeda K, Ohe Y, Kudoh S, Ichinose Y, Okamoto H, Yamamoto N, Yoshioka H, Minato K, Sawa T, Iwamoto Y, Saka H, Mizusawa J, et al. Randomized phase III trial comparing weekly docetaxel plus cisplatin versus docetaxel monotherapy every 3 weeks in elderly patients with advanced non-small-cell lung cancer: the intergroup trial JCOG0803/WJOG4307L. J Clin Oncol. 2015; 33:575-81. https://doi.org/10.1200/JCO.2014.55.8627.

38. Hironaka S, Tsubosa Y, Mizusawa J, Kii T, Kato K, Tsushima T, Chin K, Tomori A, Okuno T, Taniki T, Ura T, Matsushita H, Kojima T, et al. Phase I/II trial of 2-weekly docetaxel combined with cisplatin plus fluorouracil in metastatic esophageal cancer (JCOG0807). Cancer Sci. 2014; 105:1189-95. https://doi.org/10.1111/cas.12486.

39. Murakami H, Yamamoto N, Shibata T, Takeda K, Ichinose Y, Ohe Y, Yamamoto N, Takeda Y, Kudoh S, Atagi S, Satouchi M, Kiura K, Nogami N, et al. A single-arm confirmatory study of amrubicin therapy in patients with refractory small-cell lung cancer: Japan Clinical Oncology Group Study (JCOG0901). Lung Cancer. 2014; 84:67-72. https://doi.org/10.1016/j.lungcan.2014.01.012.

40. Miller RP, Tadagavadi RK, Ramesh G, Reeves WB. Mechanisms of Cisplatin nephrotoxicity. Toxins (Basel). 2010; 2:2490-518. https://doi.org/10.3390/toxins2112490.

41. Siddik ZH, Newell DR, Boxall FE, Harrap KR. The comparative pharmacokinetics of carboplatin and cisplatin in mice and rats. Biochem Pharmacol. 1987; 36:1925-32. 